ARTICLE

\title{
3D-3D topotactic transformation in aluminophosphate molecular sieves and its implication in new zeolite structure generation
}

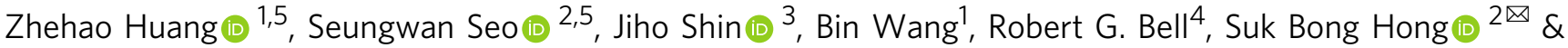 \\ Xiaodong Zou (10) ${ }^{1 \times}$
}

Zeolites have unique pore structures of molecular dimensions and tunable compositions, making them ideal for shape selective catalysis and separation. However, targeted synthesis of zeolites with new pore structures and compositions remains a key challenge. Here, we propose an approach based on a unique 3D-3D topotactic transformation, which takes advantage of weak bonding in zeolites. This is inspired by the structure transformation of PST-5, a new aluminophosphate molecular sieve, to PST-6 by calcination. The structure of nano-sized PST-5 crystals is determined by 3D electron diffraction. We find that the 3D-3D topotactic transformation involves two types of building units where penta- or hexacoordinated $\mathrm{Al}$ is present. We apply this approach to several other zeolite systems and predict a series of new zeolite structures that would be synthetically feasible. This method provides a concept for the synthesis of targeted zeolites, especially those which may not be feasible by conventional methods.

\footnotetext{
${ }^{1}$ Bezerlii Center EXSELENT on Porous Materials, Department of Materials and Environmental Chemistry, Stockholm University, SE-106 91 Stockholm, Sweden. ${ }^{2}$ Center for Ordered Nanoporous Materials Synthesis, Division of Environmental Science and Engineering, POSTECH, Pohang 37673, Korea. ${ }^{3}$ Research Center for Convergent Chemical Process, Korea Research Institute of Chemical Technology, Daejeon 34114, Korea. ${ }^{4}$ Department of Chemistry, University College London, 20 Gordon Street, London WC1H OAJ, UK. ${ }^{5}$ These authors contributed equally: Zhehao Huang, Seungwan Seo.

凶email: sbhong@postech.ac.kr; xzou@mmk.su.se
} 
Z eolites and related microporous materials have been widely used as selective catalysts, absorbents, and ionexchangers ${ }^{1-4}$. Recently zeolites have gained interest as catalysts for biomass conversion ${ }^{5-8}$ and automotive exhaust remediation ${ }^{9,10}$. The unique properties of zeolites are associated with the well-defined pores and channels of molecular dimensions. Because of the close structure-property relationship, numerous efforts have been made to synthesize zeolitic materials with new pore structures and compositions and subsequently explore their properties and applications. Currently, only 252 zeolite framework topologies have been approved by the International Zeolite Association ${ }^{11}$, even though millions of possible zeolite topologies have been identified by computational methods $^{12,13}$. The huge gap between experimental output and theoretical prediction indicates that our understanding of zeolite crystallization is still limited. It is, therefore, important to push the field by developing rational strategies to synthesize new zeolites. Besides the conventional hydro-/solvothermal synthesis, several new synthetic strategies have been proposed for targeted zeolite synthesis. These include bottom-up approaches that use, for example, pre-designed organic structure-directing agents (OSDAs) $)^{14-17}$ and promotion of specific cavities as in the synthesis of embedded isoreticular zeolites ${ }^{18,19}$, and top-down approaches, i.e., topotactic transformations. The most common top-down approach to synthesize new zeolites has been twodimensional-three-dimensional (2D-3D) layer condensation from a layered precursor ${ }^{20-23}$. Recently, a new approach of using known zeolites as precursors to synthesize new zeolites via 3D-2D-3D topotactic transformation has gained significant attention ${ }^{24-27}$. The most successful example of these methods is the assembly-disassembly-organization-reassembly (ADOR) synthesis route. The unique concept of the ADOR process is to exploit chemically selective weak $\mathrm{T}-\mathrm{O}$ bonds $(\mathrm{T}=\mathrm{Al}, \mathrm{Si}, \mathrm{Ge}$, etc.), e.g., Ge-O bonds in germanosilicates, in known zeolites and subsequently promote the formation of new $\mathrm{T}-\mathrm{O}$ bonds. The ADOR strategy provides a synthetic tool for the generation of zeolites with novel topologies and compositions that cannot be synthesized by conventional hydrothermal methods. To the best of our knowledge, these targeted synthesis strategies have only been demonstrated on silica-based zeolite syntheses (e.g., silicates, aluminosilicates and germanosilicates), but never on phosphatebased frameworks.

Aluminophosphates (known as $\mathrm{AlPO}_{4}-n$ ), first synthesized by Union Carbide ${ }^{28}$, are an important class of zeolitic molecular sieves with wide applications. $\mathrm{AlPO}_{4}$ molecular sieves have a unique feature, which allows substitution of the $\mathrm{Al}$ or $\mathrm{P}$ by main block elements (e.g., Si) or transition metals to engender specific properties in the material ${ }^{29,30}$. For example, silicoaluminophosphate SAPO-34 (framework type CHA) is one of the most widely used solid catalysts for biomass conversion, with a global market of tens of billions of US dollars per year. Currently, only $20 \%$ of the zeolite framework types can be synthesized as $\mathrm{AlPO}_{4}$ phases ${ }^{11}$, and examples of rational synthesis of $\mathrm{AlPO}_{4}$ materials are very rare ${ }^{31}$. In $\mathrm{AlPO}_{4}$ frameworks, the $\mathrm{AlO}_{4}$ and $\mathrm{PO}_{4}$ tetrahedra always alternate according to Löwenstein's rule ${ }^{32}$. Nevertheless, Al can adopt pentacoordination with a pair of $\mathrm{Al}$ atoms bridged by a hydroxyl group as found in $\mathrm{AlPO}_{4}-21(\mathrm{AWO})^{33}$ and hexa-coordination with two additional water molecules, for example, in $\mathrm{AlPO}_{4}-\mathrm{C}$ $(\mathrm{APC})^{34}$. Upon the loss of water molecules by heating, $\mathrm{AlPO}_{4}{ }^{-}$ 21 and $\mathrm{AlPO}_{4}-\mathrm{C}$ transform into new fully tetrahedrally coordinated zeolite frameworks $\mathrm{AlPO}_{4}-25$ (ATV) and $\mathrm{AlPO}_{4}-\mathrm{D}$ (APD), respectively. In 2014, we reported the synthesis and structure of a new $\mathrm{AlPO}_{4}$ zeolite denoted PST $-6^{35}$. The framework of PST-6 (PSI) has a one-dimensional (1D) pore system containing parallel 8 - and 10-ring channels. PST-6 was obtained by calcination of PST- 5 at $500^{\circ} \mathrm{C}$, which was synthesized using diethylamine (DEA) as an OSDA.

Both PST-5 and PST-6 are obtained as nano-size plate-like crystals (Supplementary Fig. 1). The structure of PST-6 was successfully solved by combining electron diffraction and powder Xray diffraction (PXRD). However, the poorer stability of PST-5 under the electron beam prevented its structure determination, and therefore the understanding of its structure transformation to PST6. Solid-state nuclear magnetic resonance (NMR) spectroscopy indicated the presence of bridging hydroxyl groups in PST-5.

Recent developments of fast 3D electron diffraction (3DED) or microcrystal electron diffraction (MicroED) data collection techniques $(<5 \mathrm{~min})$ using continuous rotation (denoted $c$ RED here) have made it possible for studying beam sensitive nano- and micrometer-sized crystals $18,36-40$. Here, we demonstrate the ab initio structure determination of PST-5 using $c$ RED data collected under low-dose condition. By detailed investigations of the structure transformation of PST-5 to PST-6, we propose an approach to generate novel zeolite structures from known zeolites via $3 \mathrm{D}-3 \mathrm{D}$ topotactic transformation. The transformation is initiated via the weak $\mathrm{Al}-\mathrm{O}$ bonding in penta-coordinated $\mathrm{AlO}_{4}(\mathrm{OH})$ pairs or hexa-coordinated $\mathrm{AlO}_{4}(\mathrm{OH})_{2}$ units. By adopting this approach, we have, in addition, predicted a series of targeted hypothetical zeolite structures.

\section{Results}

Structure of PST-5. A high-resolution $c$ RED dataset of PST-5 (with a resolution better than $0.85 \AA$ ) was collected from a submicrometer-sized crystal using low dose and fast data collection (in total $15 \mathrm{e} \AA^{-2}$ and $3 \mathrm{~min}$ ) on a JEOL JEM2100 transmission electron microscope (Fig. 1a and Supplementary Fig. 2). PST-5 crystallizes in an orthorhombic space group $P b 2 b$ (No. 27), with the unit cell parameters $a=36.5956(2) \AA, b=21.80273(9) \AA, c=$ 10.26929(4) $\AA$ (Supplementary Tables 1 and 2). Its framework structure was first solved ab initio by direct methods. To confirm the model and obtain more accurate positions and atomic displace parameters (ADPs) of individual atoms, the model was further refined against the $c$ RED data (Supplementary Table 2). Details of structure determination are given in the Supplementary Information. The framework of PST-5 exhibits a totally new topology (Supplementary Figs. 4 and 5, see Supplementary Information for more details) with a fully four-connected $3 \mathrm{D}$ net and a $2 \mathrm{D}$ pore system containing parallel 8 -ring $(5.5 \times 2.6 \AA)$ and 10 -ring $(5.8 \times 4.4 \AA)$ channels along the $c$-axis, and another 8 -ring $(3.3 \times 3.3 \AA$ ) channel along the $\boldsymbol{b}$-axis (Fig. 2). PST-5 is one of the most complex zeolite structures, with $18 \mathrm{Al}, 18 \mathrm{P}$, and $76 \mathrm{O}$ atoms in the asymmetric unit. Four of the $\mathrm{O}$ atoms belong to hydroxyl groups and act as bridging atoms to form $\mathrm{Al}-\mathrm{OH}-\mathrm{Al}$ linkages. All $18 \mathrm{P}$ atoms and 10 out of the $18 \mathrm{Al}$ atoms are tetrahedrally coordinated with $\mathrm{O}$ atom to form $\mathrm{TO}_{4}$ units $(\mathrm{T}=\mathrm{Al}, \mathrm{P})$. The remaining eight $\mathrm{Al}$ atoms have trigonal bipyramidal-coordination and form four symmetry-independent $\mathrm{AlO}_{4}-\mathrm{OH}-\mathrm{AlO}_{4}$ pairs in the structure. Each $\mathrm{AlO}_{4}-\mathrm{OH}-\mathrm{AlO}_{4}$ pair becomes part of a 3- and a 5-ring (Supplementary Fig. 6). Solid-state NMR spectroscopy confirmed the presence of Al-O-Al linkages (Supplementary Figs. 7-9), and a sharp Infrared (IR) band appearing at $3398 \mathrm{~cm}$ ${ }^{-1}$ indicates the presence of bridging $\mathrm{OH}$ groups (Supplementary Fig. 10). The structure model obtained by Rietveld refinement against synchrotron PXRD data agrees well with that obtained from $c$ RED data, which further confirms the framework structure of PST-5. In addition, the OSDA positions in the channels could be located from both $c$ RED and PXRD data, and subsequently refined by Rietveld refinement. The final refined unit cell composition is $\left.\mid\left(\mathrm{C}_{2} \mathrm{H}_{5}\right)_{2} \mathrm{NH}_{2}{ }^{+}\right)_{16} \mid\left[\mathrm{Al}_{72} \mathrm{P}_{72} \mathrm{O}_{288}\left(\mathrm{OH}^{-}\right)_{16}\right]$ (Fig. 1b, Supplementary Figs. 11 and 12 and Supplementary Table 3, 

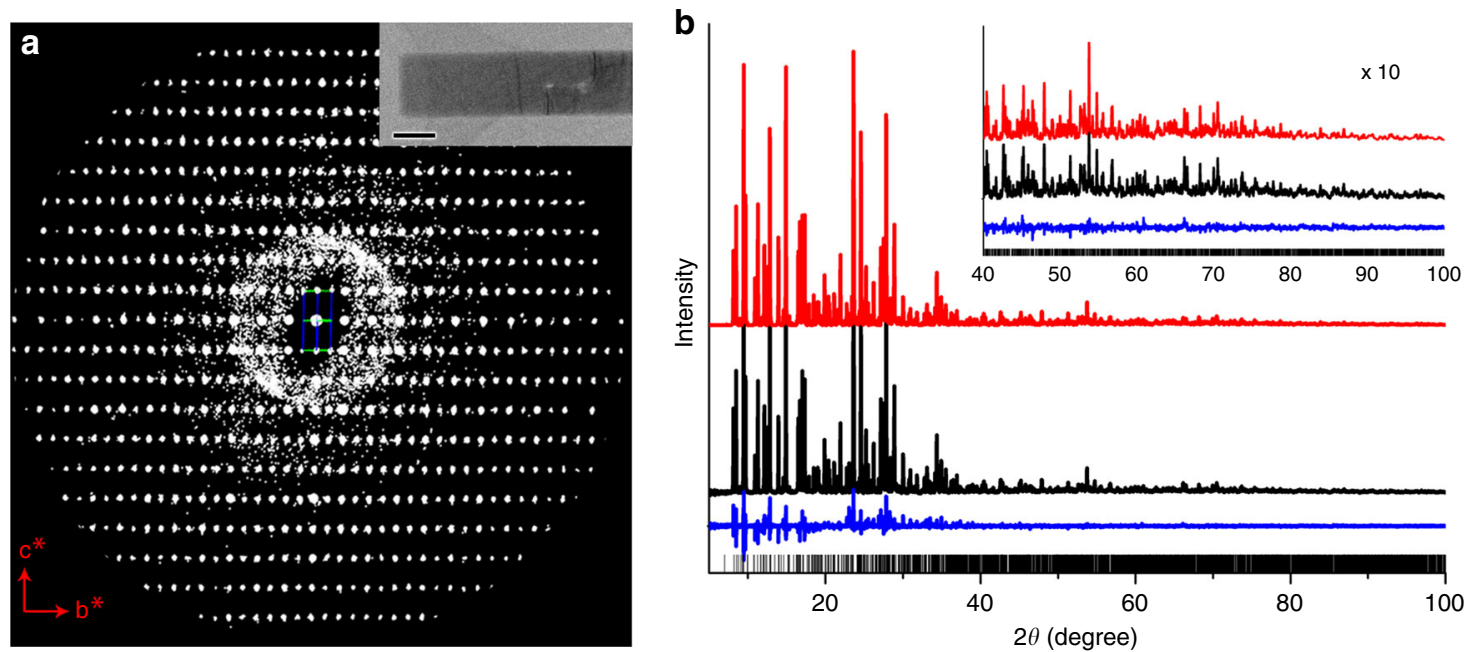

Fig. 1 Reconstructed 3D reciprocal lattice and Rietveld refinement. a Reconstructed three-dimensional reciprocal lattice of PST-5 from the continuous rotation electron diffraction (cRED) data viewed along the [100] direction. Inset is the crystal of PST-5 from which the cRED data was collected, scale bar $=$ $500 \mathrm{~nm}$. b Powder X-ray diffraction (PXRD) profiles of Rietveld refinement for PST-5 ( $\lambda=1.5474 \AA$ ): observed, calculated and difference profiles are shown in red, black, and blue, respectively. The bars represent the positions of allowed reflections.

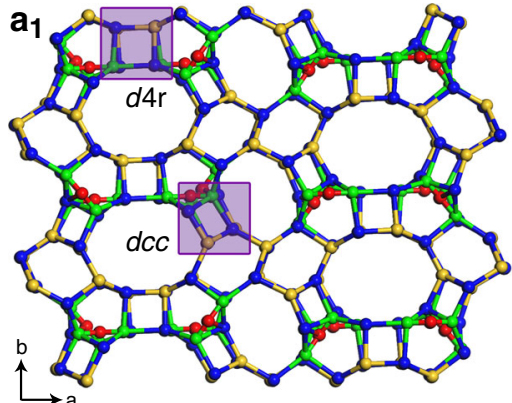

$\mathbf{a}_{2}$

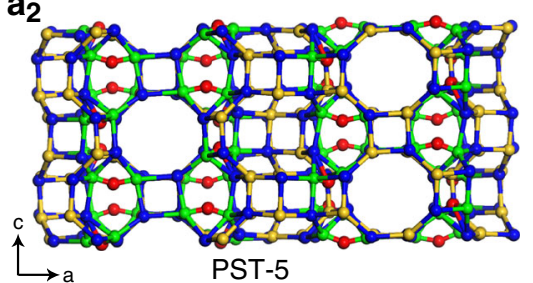

b1

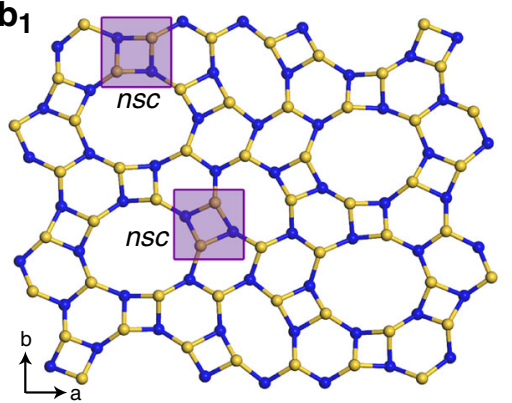

$\mathbf{b}_{2}$

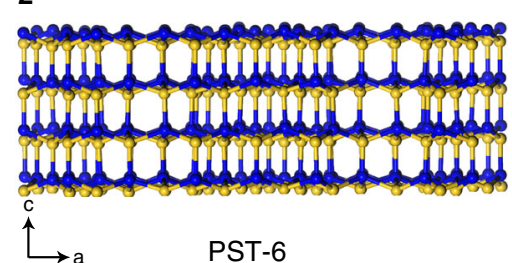

C

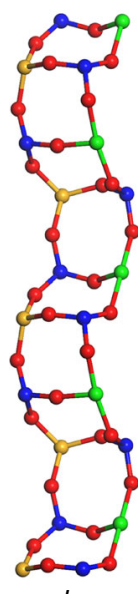

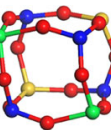

$d 4 \mathrm{r}$
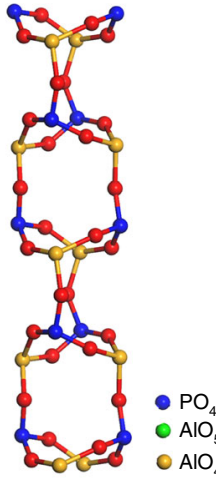

nsc 0 O

Fig. 2 Comparison of the structures of PST-5 and PST-6. Structural models of PST-5 ( $\left.\mathbf{a}_{\mathbf{1}}\right)$ and PST- $6\left(\mathbf{b}_{\mathbf{1}}\right)$ viewed along the $c-$ axis. Both structures are built from the same building layer containing 4-, 6-, 8-, and 10-rings (if the $\mathrm{OH}$ groups, shown in red are not considered). The difference is the orientations of the (Al, P) $\mathrm{O}_{4}$ tetrahedra in the two structures. The red atoms in $\left(\mathbf{a}_{\mathbf{1}}\right)$ correspond to the OH groups bridging two Al atoms in PST-5. These Al atoms are five-

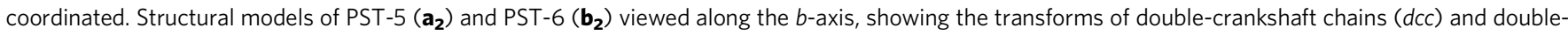
4-rings ( $d 4 r$ ) in PST-5 ( $\mathbf{a}_{\mathbf{1}}$ ) to narsarsukite-type chains (nsc) in PST-6 (indicated by purple boxes). c Building units in PST-5 (dcc and $d 4 r$ ) and PST-6 (nsc).

see Supplementary Information for more details). Raman spectrum shows that DEA molecules are protonated during PST-5 crystallization $^{35}$. Therefore, the negative framework charges created by the bridging $\mathrm{Al}-\mathrm{OH}-\mathrm{Al}$ groups are balanced by the protonated form of the occluded OSDA molecules $\left(\mathrm{DEA} \cdot \mathrm{H}^{+}\right)$, as reflected by quite similar numbers of OSDA molecules and bridging $\mathrm{O}$ atoms.

3D-3D topotactic transformation of PST-5 to PST-6. In situ PXRD patterns indicate that the structural transformation of PST-5 to PST- 6 starts at $200{ }^{\circ} \mathrm{C}$, and finishes at $500{ }^{\circ} \mathrm{C}$ (Supplementary Fig. 13), which is accompanied by notable changes in the PXRD pattern. The observed pattern after calcination of PST-5 at
$500{ }^{\circ} \mathrm{C}$ agrees very well with the simulated pattern from the PST- 6 model, confirming the calcined PST-5 (i.e., PST-6) has the PSI framework (Supplementary Fig. 14). In situ IR studies show that dehydroxylation of bridging $\mathrm{OH}$ groups in PST-5 starts above $200{ }^{\circ} \mathrm{C}$ and finishes at $300^{\circ} \mathrm{C}$ (Supplementary Fig. 10, see SI for more details). Both structures are built from a similar building layer containing 4-, 6-, 8-, and 10-rings if not considering the $\mathrm{OH}$ groups (Fig. $2 \mathrm{a}_{1}, \mathrm{~b}_{1}$ and Supplementary Fig. 15). Interestingly, the transformation of PST-5 to PST- 6 consists not only in the loss of the hydroxyl groups, as observed in the transformation of PST-13 to PST-1441. Additionally, it involves $\mathrm{T}-\mathrm{O}$ bond-breaking and reforming, which leads to totally different connections of the building layers in the two structures 

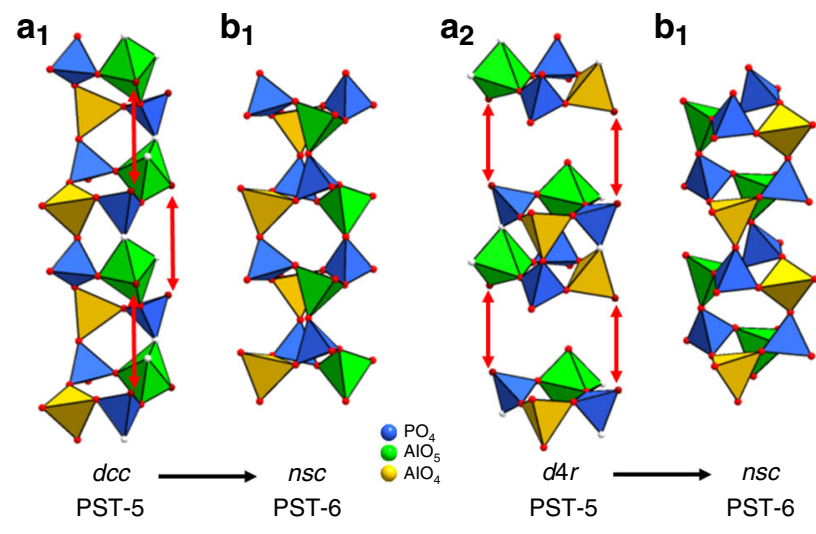

Fig. 3 Chain transformations between PST-5 and PST-6. $a_{1}$ Doublecrankshaft chain (dcc) and $\mathbf{a}_{\mathbf{2}}$ double four-ring ( $d 4 r$ ) unit in PST-5, and $\mathbf{b}_{\mathbf{1}}$ and $\mathbf{b}_{\mathbf{2}}$ narsarsukite chain (nsc) in PST-6. The removal of bridging hydroxyl groups associated with the $d c c$ and $d 4 r$ initiates the geometry changes of penta-coordinated $\mathrm{Al}$ atoms, resulting in cleavage of $\mathrm{Al}-\mathrm{O}-\mathrm{P}$ bonds (corresponding $\mathrm{O}$ atoms are shown in white) followed by inversion and reconnection of the tetrahedra (indicated by arrows). Both $d c c$ and $d 4 r$ transform to nsc in PST-6. Green, penta-coordinated (and penta-

coordinated derived) $\mathrm{AlO}_{4}$ tetrahedra; yellow, tetra-coordinated $\mathrm{AlO}_{4}$; blue, $\mathrm{PO}_{4}$. Oxygen atoms associated with $\mathrm{OH}$ or bond-breaking in the chains are shown in white and the rest $\mathrm{O}$ in red.

(Fig. $2 \mathrm{a}_{2}, \mathrm{~b}_{2}$ ) and thereby different pore systems; 1D for PST-6, in contrast to $2 \mathrm{D}$ for PST-5.

The mechanism for the 3D-3D topotactic transformation of PST-5 to PST-6 is investigated based on their structure relationships, as well as molecular dynamics simulations. PST-5 and PST6 show large structure similarities when viewed along the $\boldsymbol{c}$-axis (Fig. $2 a_{1}, b_{1}$ ). The framework of PST-5 consists mainly of two types of building units; double-crankshaft chain $(d c c)$ and double4 -ring $(d 4 r)$, both contain 4-rings, as shown in Fig. $2 c$ and highlighted in Fig. $2 \mathrm{a}_{1}$. The framework of PST-6 consists mainly of one type of building unit; a narsarsukite-type chain (nsc), as shown in Fig. $2 \mathrm{c}$ and marked in Fig. $2 \mathrm{~b}_{1}$. The transformation of PST-5 to PST- 6 can be described by the $d c c-n s c$ and $d 4 r-n s c$ transformations. It is worth noting that the $d c c-n s c$ transformation was also observed in $\mathrm{AlPO}_{4}-21$ to $\mathrm{AlPO}_{4}-25^{33}$, and $\mathrm{AlPO}_{4}-\mathrm{C}$ to $\mathrm{AlPO}_{4}-\mathrm{D}^{34}$. However, the $d 4 r$-nsc transformation has not been reported before.

The key to the chain transformation is the penta-coordinated $\mathrm{Al}$ atoms, where $\mathrm{Al}-\mathrm{O}$ bond lengths (on average 1.83(9) $\AA$ ) are longer than those of tetra-coordinated $\mathrm{Al}$ atoms (on average 1.72 (1) $\AA$ ). The longer bond lengths indicate weaker bonds, which are more easily broken. Therefore, the built-in weakness associated with penta-coordinated $\mathrm{Al}$ atoms in the structure may be utilized to initiate the topotactic transformation. Upon thermal treatment, the bridging hydroxyl groups are removed through a dehydroxylation to form the fully four-connected framework PST-6 (Fig. 2 and Supplementary Fig. 6).

By detailed inspection of the structural relationships between PST-5 and PST-6, the geometry changes of penta-coordinated Al atoms initiate a domino geometrical inversion of nearby $\mathrm{PO}_{4}$ tetrahedra. Upon heating, the penta-coordinated $\mathrm{Al}$ atoms lose the bridging hydroxyl groups, which also leads to cleavage of the $\mathrm{Al}-\mathrm{O}-\mathrm{P}$ bond to the adjacent $\mathrm{PO}_{4}$ tetrahedra. The $\mathrm{AlO}_{4}$ and $\mathrm{PO}_{4}$ tetrahedral pairs with resulting broken bonds then invert their orientations and reconnect to the next nearest pairs of $\mathrm{AlO}_{4}$ and $\mathrm{PO}_{4}$ tetrahedra (Fig. 3, indicated by arrows). As a result, the double-crankshaft chains $(d c c)$ in PST-5 transforms to the narsarsukite-type chains ( $n s c$ ) in PST-6, which is composed of one chain with tetra-coordinated $\mathrm{Al}$ atoms, and another chain originating from penta-coordinated $\mathrm{Al}$ atoms (Fig. $3 \mathrm{a}_{1}, \mathrm{~b}_{1}$ ). The inversion further extends to neighboring atoms, which results in a domino-like alteration of the tetrahedral conformation. There are two symmetry-independent $d c c$ chains in PST-5 with different orientations. After being transformed to PST-6, they orient in the same way. The reconstructed PST-6 (PSI) material was found to contain stacking faults ${ }^{35}$, due to changes in both orientation and tetrahedral conformation. There are eight unique pentacoordinated $\mathrm{Al}$ atoms that can initiate the topotactic transformation. When the transformation starts at different positions, it will also generate disorder after the reconstruction.

To study the possible mechanisms for the transformation of PST-5 to PST-6, periodic ab initio molecular dynamics simulations were carried out on a model PST-5 system. In nearly every case, one bond from penta-coordinated Al was broken, forming tetra-coordinated $\mathrm{Al}$ and corresponding $\mathrm{P}-\mathrm{OH}$ groups (Supplementary Figs. 16 and 17). In one case, the Al-O-P linkage was broken within a $3-(-\mathrm{Al}-\mathrm{O}-\mathrm{P}-\mathrm{Al}-\mathrm{OH}-)$ ring, but otherwise the framework interruption occurred between the penta-coordinated $\mathrm{Al}$ and neighboring $\mathrm{P}$ atoms, which were part of a doublecrankshaft chain $(d c c)$ or a double-4-ring $(d 4 r)$ structural unit but not in a 3-ring. The simulations clearly confirm that weaker bonds in the PST-5 framework are associated with pentacoordinated $\mathrm{Al}$ atoms and are labile in the presence of an extraframework proton donor. The initial broken bonds are those in the $d c c$ and $d 4 r$, largely the same as those implicated in the transformation of PST-5 to PST-6 at the experimental annealing temperature. Coupled with the topological strain inherent within the $d c c$ and $d 4 r$ due to their fused 4-ring motifs, the pentacoordinated $\mathrm{Al}$ atoms are thus critical in introducing reactivity into the framework. It should be noted that the molecular dynamics simulations of an idealized, fully four-connected, $\mathrm{AlPO}_{4}$ PST-5 framework, without penta-coordinated $\mathrm{Al}$ atoms and bridging $\mathrm{OH}$ groups, showed no cleavage of any framework bonds over the same timescale at $500{ }^{\circ} \mathrm{C}$.

New zeolite generation. Inspired by the 3D-3D topotatic transformation in PST-5/PST-6, we investigated the existing structures in the Zeolite Structure Database ${ }^{11}$. 12 framework types are found to contain infinite $d c c$, and they can be divided into three groups based on the structural complexity (Table 1): I) built solely from $d c c$ that are connected directly (APC, GIS, MER, PHI, SIV, GME, Supplementary Fig. 18); II) built from $d c c$ that are bridged by isolated 4-rings (ATT, AWO, UEI, Supplementary Figs. 19 and 20); III) built by $d c c$ and other building units (PST-5, DON, STO, Supplementary Fig. 21). Interestingly, regardless of the complexity, the $d c c-n s c$ structural transformation has been observed in all groups, from $\mathrm{AlPO}_{4}-\mathrm{C}(\mathrm{APC})$ to $\mathrm{AlPO}_{4}-\mathrm{D}$ (APD) in group $\mathrm{I}, \mathrm{AlPO}_{4}-21$ (AWO) to $\mathrm{AlPO}_{4}-25$ (ATV) in group II, and PST-5 to PST-6 (PSI) in group III (Table 1 and Supplementary Table 4). The three parent frameworks all contain dcc in which penta- $\left(\mathrm{AlPO}_{4}-21\right.$ and PST-5) or hexa- $\left(\mathrm{AlPO}_{4}-\mathrm{C}\right)$ coordinated $\mathrm{Al}$ atoms are present. By applying similar dcc to nsc transformations observed in the three known zeolite pairs to other $d c c$-containing zeolites, we generated a series of novel hypothetical $n s c$-containing zeolite structures, as shown in Fig. 4, Supplementary Figs. 18-22, Table 1, and Supplementary Table 5 (see Supplementary Information for more details) ${ }^{42}$. Notably, the same parent zeolite can generate more than one hypothetical zeolites (e.g., UEI to UEI_H1-5), and the same zeolite can be generated from different parent zeolites (e.g., GIS_H1 from both GIS and SIV). The transformation can also generate new channel dimensionality (Supplementary Fig. 18 and Table 1). All these hypothetical $n s c$-containing zeolites fulfill the criteria for zeolite frameworks realizable for target synthesis (Supplementary 


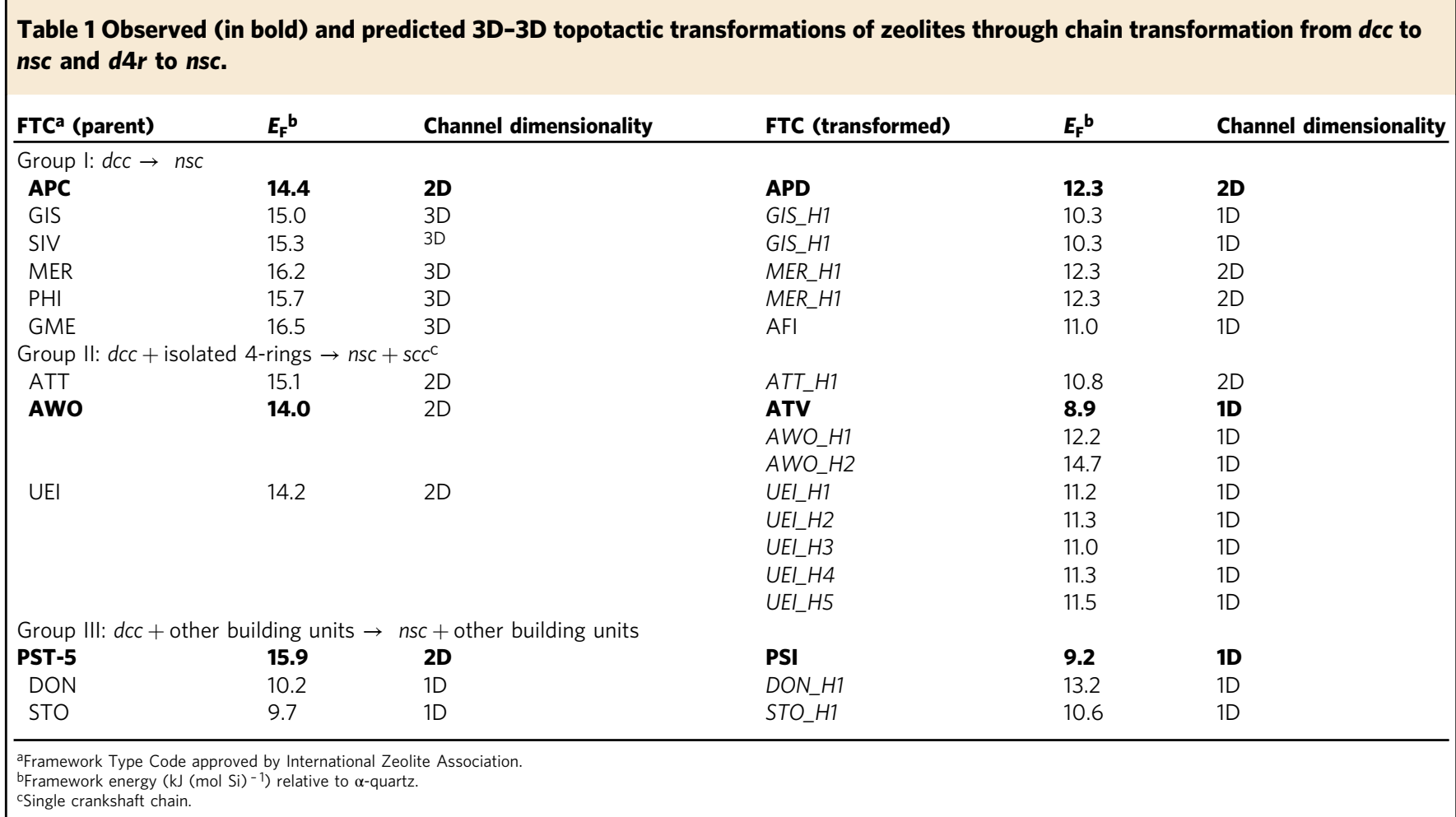

Table 6$)^{43}$. Most of them were calculated to have much lower framework energies than the parent $d c c$-containing zeolites. It is worth mentioning that because each $\mathrm{TO}_{4}$ tetrahedral unit not belonging to $d c c$ can have two possible connectivities, either upwards or downwards, the number of hypothetical zeolites generated via this approach can be enormous for zeolites in group II and III (see Supplementary Information for more details).

We have demonstrated that many hypothetical zeolites can be predicted from known zeolite frameworks based on the $d c c-n s c$ transformation. The 3D-3D topotactic transformation would provide a simple approach to generate novel zeolite frameworks, which may be inaccessible by conventional hydrothermal/ solvothermal synthesis. We have also shown by molecular dynamics simulations that the weakened bonding, such as pentaor hexa-coordinated $\mathrm{Al}$ atoms, is crucial to initiate the $3 \mathrm{D}-3 \mathrm{D}$ topotactic transformation. Therefore, to synthesize new structures by this approach, it is important to introduce penta- or hexacoordinated $\mathrm{Al}$ atoms in $d c c$-containing zeolite frameworks, and additional exploratory work is needed. Previous studies showed that certain types of OSDAs, like easily protonatable amines, could promote the formation of bridging $\mathrm{Al}-\mathrm{OH}-\mathrm{Al}$ bonds in $\mathrm{AlPO}_{4}$ molecular sieves, and penta- or hexa-coordinated $\mathrm{Al}$ atoms have been found in c.a. $25 \%$ of these materials, including $\mathrm{AlPO}_{4}$ 17, $\mathrm{AlPO}_{4}-41, \mathrm{AlPO}_{4}$-EN3, AlPO-CJB1, VPI-5, IST-1, PST-13, etc. ${ }^{41,44-50}$. The $3 \mathrm{D}-3 \mathrm{D}$ topotactic transformation may also be applicable to other zeolite compositions. For example, the gallophosphate $\mathrm{Mu}-18$ (UEI) contains both $d c c$ and pentacoordinated atoms (Fig. $\left.4 a_{7}\right)^{51}$. We anticipate that with the discovery of many new zeolites, the approach described here will be more feasible.

\section{Discussion}

We have solved the structure of PST-5, a new 2D medium-pore $\mathrm{AlPO}_{4}$ molecular sieve, using continuous rotation electron diffraction ( $c \mathrm{RED})$. Based on the investigations of the $d c c$ to $n s c$ transformations observed in PST-5/PST-6, $\mathrm{AlPO}_{4}-\mathrm{C} / \mathrm{AlPO}_{4}-\mathrm{D}$, and $\mathrm{AlPO}_{4}-21 / \mathrm{AlPO}_{4}-25$ pairs, we propose an approach to generate novel zeolite structures, which enriches the structural diversity. The 3D-3D topotactic transformation relies on the chemical weakness in the $d c c$. PST-5 transforms $d c c$ and $d 4 r$ to $n s c$, changes its overall interlayer connectivities and finally generates PST-6. The penta-coordinated $\mathrm{Al}$ atoms, as observed in PST-5, could be built into a precursor material by using predesigned OSDAs, which balance the framework energy. This approach via the $3 \mathrm{D}-3 \mathrm{D}$ topotactic transformation can be considered complementary to the existing strategies of synthesizing novel zeolite structures. By understanding the 3D-3D topotactic transformation, we anticipate that our approach can be generalized and provide new insights into the discovery of novel zeolitic materials, which may enable a completely new range of zeolite frameworks to be accessible and offer opportunities in technological applications.

\section{Methods}

Synthesis of PST-5. The reagents used for the synthesis of PST-5 included diethylamine (DEA, $99.5+\%$, Aldrich), aluminum isopropxide (98+\%, Aldrich), phosphoric acid ( $85 \%$, Merck), and deionized water. PST-5 was synthesized from an $\mathrm{AlPO}_{4}$ gel with the composition of $2.0 \mathrm{DEA} \cdot 1.0 \mathrm{Al}_{2} \mathrm{O}_{3} \cdot 1.0 \mathrm{P}_{2} \mathrm{O}_{5} \cdot 40 \mathrm{H}_{2} \mathrm{O}$, where DEA is diethylamine. In a typical synthesis of PST-5, $3.84 \mathrm{~g}$ of $o-\mathrm{H}_{3} \mathrm{PO}_{4}(85 \%$, Merck) was diluted in $5.63 \mathrm{~g}$ of $\mathrm{H}_{2} \mathrm{O}$. This solution was added dropwise to a slurry of $6.95 \mathrm{~g}$ of aluminum isopropoxide ( $>98 \%$, Aldrich) in $5.64 \mathrm{~g}$ of $\mathrm{H}_{2} \mathrm{O}$. After stirring for $1 \mathrm{~h}, 2.45 \mathrm{~g}$ of DEA (99.5\%, Aldrich) was added to the above synthesis mixture, which was stirred overnight at room temperature. Afterwards, the mixture was charged into a Teflon-lined 23-mL autoclave and heated under static conditions at $200^{\circ} \mathrm{C}$ for 5 days. The resulting white powder was recovered by filtration, washed repeatedly with water and then dried overnight at room temperature.

Topotactic transformation of PST-5. The topotactic transformation of PST-5 to PST -6 was conducted by calcination of PST -5 at $550^{\circ} \mathrm{C}$ for $8 \mathrm{~h}$ under air flow.

CRED data collection. PST-5 powder in its as-made form was dispersed in ethanol and a droplet of the suspension was transferred onto a carbon-coated copper grid and dried in air. $c$ RED data was acquired in selected-area mode with a goniometer tilt speed of $0.45^{\circ} \mathrm{s}^{-1}$ and an exposure time of $0.4 \mathrm{~s}$ per frame (Supplementary Table 2). The total data collection time was $<3 \mathrm{~min}$ in order to minimize the beam damage and maximize the data quality. The total tilt range was $92.6^{\circ}$. 

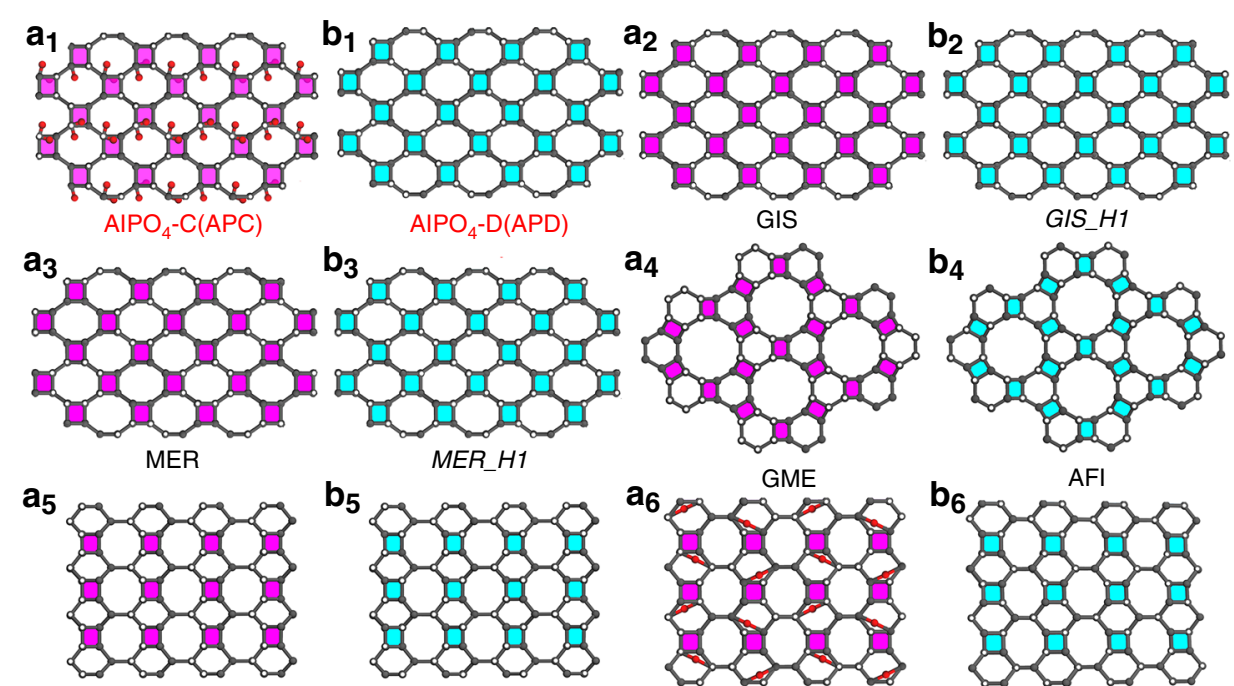

$\mathbf{b}_{5}$
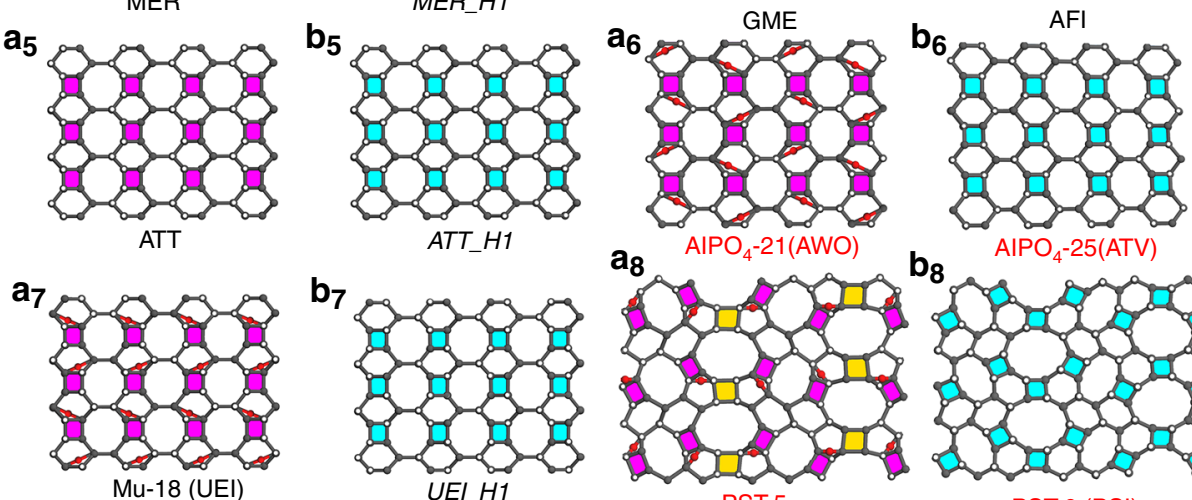

$\mathbf{b}_{7}$
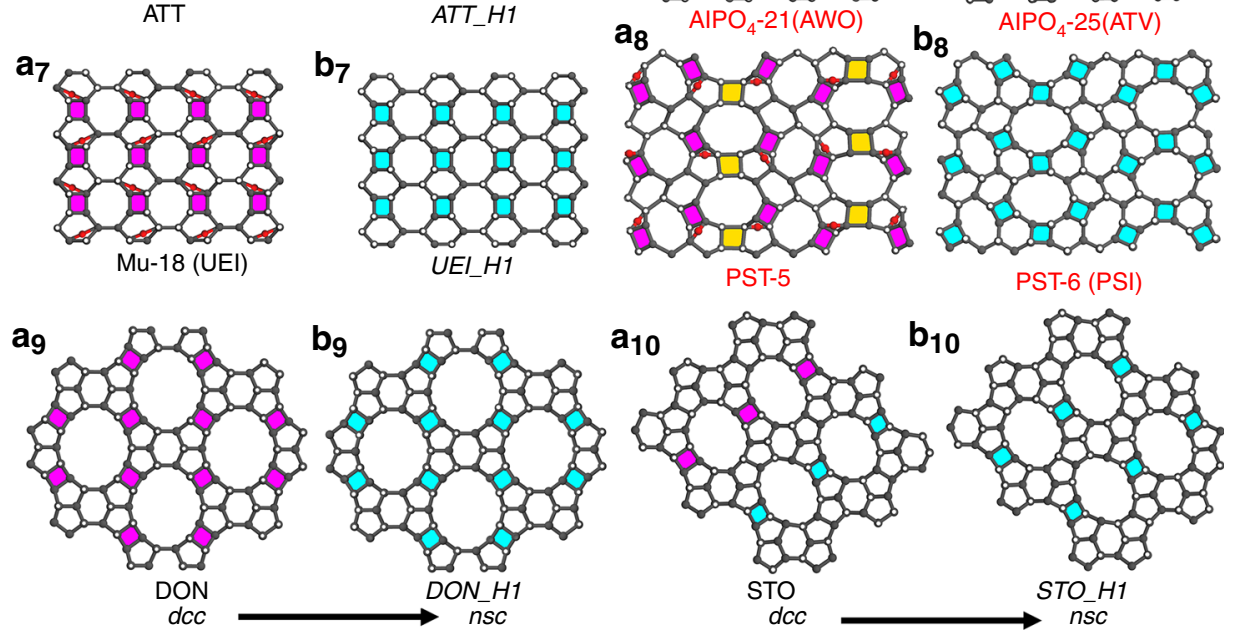

Fig. 4 Illustration of observed and predicted 3D-3D topotactic transformations. $\mathbf{a}_{\mathbf{1}}-\mathbf{a}_{\mathbf{1 0}}$ Projections of the APC, GIS, MER, GME, ATT, AWO, UEI, PST5, DON, and STO frameworks. All of them contain double-crankshaft chain (dcc). Among them, APC, AWO, UEI, and PST-5 contain penta- or hexacoordinated atoms. $\mathbf{b}_{\mathbf{1}}, \mathbf{b}_{\mathbf{6}}$, and $\mathbf{b}_{\mathbf{8}}$ Projections of APD, ATV, and PSI (PST-6) frameworks after topotactic transformation by changing dcc to narsarsukite chain (nsc). They have the same framework projections as APC, AWO, and PST-5, respectively, but differ in the 3D connectivity along the projections. $\mathbf{b}_{\mathbf{2}}-\mathbf{b}_{\mathbf{5}}, \mathbf{b}_{\mathbf{7}}, \mathbf{b}_{\mathbf{9}}$, and $\mathbf{b}_{\mathbf{1}}$ Selected hypothetical structures of GIS_H1, MER_H1, ATT_H1, UEI_H1, DON_H1, and STO_H1 generated by replacing $d c c$ with nsc. Magenta: $d c c$; cyan: nsc; yellow: double four-ring $(d 4 r)$. The solid and hollow circles indicate vertices that connect upwards and downwards, respectively.

\section{Data availability}

The crystallographic information files for PST-5 refined against the cRED and PXRD data have been deposited at the Cambridge Crystallographic Data Center (CCDC, free for charge at https://www.ccdc.cam.ac.uk) under deposition number CCDC 1948775 and 1944918, respectively. The cif containing all the hypothetical structures and Supplementary information are available in the online version of the paper. Reprints and permissions information is available online at www.nature.com/reprints. Correspondence and requests for materials should be addressed to S.B.H. (sbhong@postech.ac.kr) and X.Z. (xzou@mmk.su.se).

Received: 9 January 2020; Accepted: 6 July 2020;

Published online: 28 July 2020

\section{References}

1. Climent, M. J., Corma, A. \& Iborra, S. Heterogeneous catalysts for the one-pot synthesis of chemicals and fine chemicals. Chem. Rev. 111, 1072-1133 (2011).

2. Chen, L.-H. et al. Hierarchically structured zeolites: synthesis, mass transport properties and applications. J. Mater. Chem. 22, 17381-17403 (2012).

3. Dusselier, M. \& Davis, M. E. Small-pore zeolites: synthesis and catalysis. Chem. Rev. 118, 5265-5329 (2018).
4. Datta, S. J. et al. $\mathrm{CO}_{2}$ capture from humid flue gases and humid atmosphere using a microporous coppersilicate. Science 350, 302-306 (2015).

5. Yarulina, I., Chowdhury, A. D., Meirer, F., Weckhuysen, B. M. \& Gascon, J. Recent trends and fundamental insights in the methanol-to-hydrocarbons process. Nat. Catal. 1, 398 (2018).

6. Li, Y., Li, L. \& Yu, J. Applications of zeolites in sustainable chemistry. Chem 3, 928-949 (2017).

7. Lee, J. H. et al. Synthesis and characterization of ERI-type UZM-12 zeolites and their methanol-to-olefin performance. J. Am. Chem. Soc. 132, 12971-12982 (2010).

8. Sudarsanam, P., Peeters, E., Makshina, E. V., Parvulescu, V. I. \& Sels, B. F. Advances in porous and nanoscale catalysts for viable biomass conversion. Chem. Soc. Rev. 48, 2366-2421 (2019).

9. Marberger, A. et al. Time-resolved copper speciation during selective catalytic reduction of NO on Cu-SSZ-13. Nat. Catal. 1, 221 (2018).

10. Zhang, L. et al. Recent advances in the preparation of zeolites for the selective catalytic reduction of NOx in diesel engines. React. Chem. Eng. 4, 975-985 (2019).

11. Baerlocher, C. \& McCusker, L. B. Database of Zeolite Structures. http://www. iza-structure.org/databases/ (accessed in May 20, 2020).

12. Akporiaye, D. E. \& Price, G. D. Systematic enumeration of zeolite frameworks. Zeolites 9, 23-32 (1989).

13. Pophale, R., Cheeseman, P. A. \& Deem, M. W. A database of new zeolite-like materials. Phys. Chem. Chem. Phys. 13, 12407-12412 (2011). 
14. Moliner, M., Rey, F. \& Corma, A. Towards the rational design of efficient organic structure-directing agents for zeolite synthesis. Angew. Chem. Int. Ed. 52, 13880-13889 (2013).

15. Gallego, E. M. et al. "Ab initio" synthesis of zeolites for preestablished catalytic reactions. Science 355, 1051-1054 (2017)

16. Li, Y., Li, X., Liu, J., Duan, F. \& Yu, J. In silico prediction and screening of modular crystal structures via a high-throughput genomic approach. Nat. Commun. 6, ncomms9328 (2015).

17. Park, M. B., Cho, S. J. \& Hong, S. B. Synthesis of aluminosilicate and gallosilicate zeolites via a charge density mismatch approach and their characterization. J. Am. Chem. Soc. 133, 1917-1934 (2011).

18. Guo, P. et al. A zeolite family with expanding structural complexity and embedded isoreticular structures. Nature 524, 74-78 (2015).

19. Shin, J. et al. Targeted synthesis of two super-complex zeolites with embedded isoreticular structures. Angew. Chem. Int. Ed. 55, 4928-4932 (2016).

20. Leonowicz, M. E., Lawton, J. A., Lawton, S. L. \& Rubin, M. K. MCM-22: a molecular sieve with two independent multidimensional channel systems. Science 264, 1910-1913 (1994).

21. Zanardi, S. et al. Crystal structure determination of zeolite $\mathrm{Nu}-6(2)$ and its layered precursor Nu-6(1). Angew. Chem. Int. Ed. 43, 4933-4937 (2004).

22. Ikeda, T., Akiyama, Y., Oumi, Y., Kawai, A. \& Mizukami, F. The topotactic conversion of a novel layered silicate into a new framework zeolite. Angew. Chem. Int. Ed. 43, 4892-4896 (2004).

23. Wang, Y. X., Gies, H., Marler, B. \& Müller, U. Synthesis and crystal structure of zeolite RUB-41 obtained as calcination product of a layered precursor: a systematic approach to a new synthesis route. Chem. Mater. 17, 43-49 (2005).

24. Roth, W. J. et al. A family of zeolites with controlled pore size prepared using a top-down method. Nat. Chem. 5, 628-633 (2013).

25. Mazur, M. et al. Synthesis of 'unfeasible' zeolites. Nat. Chem. 8, 58-62 (2016).

26. Verheyen, E. et al. Design of zeolite by inverse sigma transformation. Nat. Mater. 11, 1059-1064 (2012)

27. Marler, B. \& Gies, H. Hydrous layer silicates as precursors for zeolites obtained through topotactic condensation: a review. Eur. J. Miner. 24, 405-428 (2012).

28. Wilson, S. T., Lok, B. M., Messina, C. A., Cannan, T. R. \& Flanigen, E. M. Aluminophosphate molecular sieves: a new class of microporous crystalline inorganic solids. J. Am. Chem. Soc. 104, 1146-1147 (1982).

29. Yu, J. \& Xu, R. Insight into the construction of open-framework aluminophosphates. Chem. Soc. Rev. 35, 593-604 (2006).

30. Cheetham, A. K., Férey, G. \& Loiseau, T. Open-framework inorganic materials. Angew. Chem. Int. Ed. 38, 3268-3292 (1999).

31. Broach, R. W. et al. New ABC- 6 net molecular sieves ZnAPO-57 and ZnAPO59: framework charge density-induced transition from two- to threedimensional porosity. Micropor. Mesopor. Mater. 189, 49-63 (2014).

32. Loewenstein, W. The distribution of aluminum in the tetrahedra of silicates and aluminates. Am. Min. 39, 92-96 (1954).

33. Richardson, J. W., Smith, J. V. \& Pluth, J. J. AlPO 4 -25: framework topology, topotactic transformation from AlPO4-21, and high-low displacive transition. J. Phys. Chem. 94, 3365-3367 (1990).

34. Keller, E. B., Meier, W. M. \& Kirchner, R. M. Synthesis, structures of $\mathrm{AlPO}_{4}-\mathrm{C}$ and $\mathrm{AlPO}_{4}$-D, and their topotactic transformation. Solid State Ion. 43, 93-102 (1990).

35. Lee, J. K. et al. An aluminophosphate molecular sieve with 36 crystallographically distinct tetrahedral sites. Angew. Chem. Int. Ed. 53 7480-7483 (2014).

36. Warren, M. 'Why didn't we think to do this earlier?' Chemists thrilled by speedy atomic structures. Nature 563, 16 (2018).

37. Brázda, P., Palatinus, L. \& Babor, M. Electron diffraction determines molecular absolute configuration in a pharmaceutical nanocrystal. Science 364, 667-669 (2019).

38. Sun, J. et al. The ITQ-37 mesoporous chiral zeolite. Nature 458, 1154-1157 (2009).

39. Yuan, S. et al. $\left[\mathrm{Ti}_{8} \mathrm{Zr}_{2} \mathrm{O}_{12}(\mathrm{COO})_{16}\right]$ Cluster: An Ideal Inorganic Building Unit for Photoactive Metal-Organic Frameworks. ACS Cent. Sci. 4, 105-111 (2018).

40. Huang, Z. et al. Can 3D electron diffraction provide accurate atomic structures of metal-organic frameworks? Faraday Discuss. https://doi.org/10.1039/ D0FD00015A (2020)

41. Seo, S. et al. Two aluminophosphate molecular sieves built from pairs of enantiomeric structural building units. Angew. Chem. Int. Ed. 57, 3727-3732 (2018).

42. Foster, M. D. \& Treacy, M. M. A Database of Hypothetical Zeolite Structures. http://www.hypotheticalzeolites.net/ (accessed May 20, 2020).

43. Li, Y., Yu, J. \& Xu, R. Criteria for zeolite frameworks realizable for target synthesis. Angew. Chem. Int. Ed. 52, 1673-1677 (2013).

44. Parise, J. B. In Studies in Surface Science and Catalysis (eds Držaj, B., Hočevar, S. \& Pejovnik, S.) Vol. 24 271-278 (Elsevier, 1985)
45. Hartmann, M., Prakash, B. M. \& Kevan, L. Multinuclear MAS NMR study on the microporous aluminophosphates $\mathrm{AlPO}_{4}-41$ and SAPO-41. J. Chem. Soc. Faraday Trans. 94, 723-727 (1998)

46. Yan, W. et al. $\left[\mathrm{Al}_{12} \mathrm{P}_{13} \mathrm{O}_{52}\right]^{3-}\left[\left(\mathrm{CH}_{2}\right)_{6} \mathrm{~N}_{4} \mathrm{H}_{3}\right]^{3+}$ : An anionic aluminophosphate molecular sieve with brönsted acidity. Chem. Mater. 12, 2517-2519 (2000)

47. Davis, M. E., Saldarriaga, C., Montes, C., Garces, J. \& Crowdert, C. A molecular sieve with eighteen-membered rings. Nature 331, 698-699 (1988).

48. Broach, R. W., Wilson, S. T. \& Kirchner, R. M. Corrected crystallographic tables and figure for as-synthesized $\mathrm{AlPO}_{4}$-14. Micropor. Mesopor. Mater. 57, 211-214 (2003)

49. Jordá, J. L. et al. Structure analysis of the novel microporous aluminophosphate IST-1 using synchrotron powder diffraction data and HETCOR MAS NMR. Micropor. Mesopor. Mater. 65, 43-57 (2003)

50. Pluth, J. J., Smith, J. V. \& Bennett, J. M. Microporous aluminophosphate number 17 with encapsulated piperidine, topological similarity to erionite. Acta Crystallogr. C. 42, 283-286 (1986).

51. Josien, L., Simon, A., Gramlich, V. \& Patarin, J. Synthesis and characterization of the new hydroxygallophosphate $\mathrm{Mu}-18$ with a framework topology closely related to that of the hydroxyaluminophosphate $\mathrm{AlPO}_{4}$-EN3. Chem. Mater. 13, 1305-1311 (2001)

\section{Acknowledgements}

This work was supported by the Swedish Research Council (VR, 2017-04321, 201604625), the Knut and Alice Wallenberg Foundation (KAW, 2012-0112) through the 3DEM-NATUR project and National Creative Research Initiative Program (2012R1A3A2048833) through the National Research Foundation of Korea and the National Research Council of Science \& Technology (CRC-14-1-KRICT) grant by the Korea government (MSIP). We thank PAL (Pohang, Korea) for synchrotron diffraction beam time at beamline 9B (D. Ahn). PAL is supported by MSIP and POSTECH. We also thank G.J. Kennedy (ExxonMobil) for the ${ }^{27} \mathrm{Al} 3 \mathrm{Q}$ MAS and ${ }^{27} \mathrm{Al} \rightarrow{ }^{31} \mathrm{P}$ 3Q HETCOR NMR measurements. Open access funding provided by Stockholm University.

\section{Author contributions}

Original idea was conceived by X.Z., S.B.H., Z.H., and S.S.; synthesis work was performed by S.S. and J.S., structural characterization was performed by Z.H. and B.W.; Rietveld refinement was performed by S.S. and J.S.; data analysis was performed by Z.H., S.S., and J.S.; molecular simulations were performed by R.B.; new structure generation was performed by Z.H. and S.S.; manuscript was drafted by Z.H., S.S., X.Z., S.B.H., R.B., J.S., and B.W. All authors have given approval to the manuscript.

\section{Competing interests}

The authors declare no competing interests.

\section{Additional information}

Supplementary information is available for this paper at https://doi.org/10.1038/s41467020-17586-7.

Correspondence and requests for materials should be addressed to S.B.H. or X.Z.

Peer review information Nature Communications thanks Yu Han and other, anonymous, reviewers for their contributions to the peer review of this work. Peer review reports are available.

Reprints and permission information is available at http://www.nature.com/reprints

Publisher's note Springer Nature remains neutral with regard to jurisdictional claims in published maps and institutional affiliations.

pen Access This article is licensed under a Creative Commons Attribution 4.0 International License, which permits use, sharing, adaptation, distribution and reproduction in any medium or format, as long as you give appropriate credit to the original author(s) and the source, provide a link to the Creative Commons license, and indicate if changes were made. The images or other third party material in this article are included in the article's Creative Commons license, unless indicated otherwise in a credit line to the material. If material is not included in the article's Creative Commons license and your intended use is not permitted by statutory regulation or exceeds the permitted use, you will need to obtain permission directly from the copyright holder. To view a copy of this license, visit http://creativecommons.org/ licenses/by/4.0/.

(C) The Author(s) 2020 